\title{
XLIX. On the appreciation of ultra-visible quantities, and on a gauge to help us to appreciate them
}

\section{G. Johnstone Stoney M.A. D.Sc. F.R.S.}

To cite this article: G. Johnstone Stoney M.A. D.Sc. F.R.S. (1892) XLIX. On the appreciation of ultra-visible quantities, and on a gauge to help us to appreciate them , Philosophical Magazine Series 5, 34:210, 415-429, DOI: 10.1080/14786449208620354

To link to this article: http://dx.doi.org/10.1080/14786449208620354

曲 Published online: 08 May 2009.

Submit your article to this journal $[\pi$

Џll Article views: 2

Q View related articles $₫$ 
XLIX. On the Appreciation of Ultra-visible Quantities, and on a Gauge to help us to appreciate them. By G. JoHNStone Stoner, M.A., D.Sc., F.R.S., Vice-President, Royal Jublin Society*.

\section{Description of the Gauge.}

TMAGINE a quadrant of the earth's meridian to be 1 straightened out, and used as the base-line of a wedgeshaped gauge. Set a metre upright at one end of this base, and from the top of it draw the inclined plane to the other end. This completes the gauge. It is, in fact, a wedge with a slope of one in ten millions. We shall only require the last ten metres of this gauge, next its apex; and it is this portion which I propose as a standard for the measurement of small quantities. Small quantities are to be measured by the ordinates of the gauge, that is by the little perpendicular distances from its base-line up to its sloping top.

Another and perhaps a better way of conceiving the gauge is to take a base-line that is only ten metres long, to erect a micront at one end, and from the top of this to draw the incline to the other end. This will give the same slope as before-a gradient of one in 10,000,000.

\section{Illustrations of the very Acute Angle of this Gauge.}

1. A wedge with an angle of $1^{\prime \prime}$ would furnish a slope of one in 206,265. Ours has a slope of only one in 10,000,000. It is, accordingly, between 48 and 49 times more acute: in other words, its angle is less than the forty-eighth of $1^{\prime \prime}$, which is a much smaller angle than can be measured by any astronomical instrument.

2. Prolong the gauge beyond the ten metres. Then the slightly differing diameters of the red corpuscles in human blood are equal to the ordinates of the gauge at from 70 to 80 metres from its apex-about as far as street-lamps are from noe another.

3. At 10 kilometres distance (over six miles) the ordinate is exactly one millimetre.

* From the 'Scientific Proceedings' of the Royal Dublin Society, vol. vii. p. 530. Communicated by the Author.

+ The micron is a measure that has come of late years into general use among microscopists. It is the thousandth of a millimetre, which is the same as 1/25400th of an inch. The micron is between the seventh and the eighth part of the diameters of the little red corpuscles in human blood, which are tolerably uniform in size and are familiar objects to all worliers with the microscope. 
4. And to reach an ordinate which is as long as an inch, we should have to go to a distance of 254 kilometres from the apex-about 158 miles, or across Ireland.

However, in the study of molecular physics, we are dealing with measures that are fractions of a micron; so that the ten metres of our gauge that are next its apex are enough for us to retain.

\section{Relation of the Gauge to Ångström's Map.}

The wave-lengths of light are the longest of the small quantities with which we need concern ourselves; and the gradient of the gauge has been specially chosen to be convenient in measuring them. For this purpose, lay the gauge on Ångström's map of the "Spectre normal du Soleil," making the points on the base-line at 4, 5, and 6 metres from the apex of the gange coincide with the positions 4000, 5000 , and 6000 on his map. This can be done, since Angström's scale is a scale of millimetres.

Then the actual length of $\lambda$ (the wave-length in air) for each ray represented in his map, is the ordinate of the gauge (i. e. the vertical distance from the horizontal base of the gauge up to its sloping top) immediately over the line of the map representing the ray*.

\section{The "Minimum visibile."}

The minimum visibile (i.e. the smallest separation at which two points must stand to admit of their being seen as two, by the help of such coarse waves as the waves of light) is about half the wave-length of the light admitted to our microscope; that is, it is the ordinate of our standard gauge at some point between two and three metres from its apex. All smaller magnitudes are ultra-visible.

\section{The Larger Ultra-visible Magnitudes.}

1. Ponderable matter is in the gaseous state when its molecules are so little crowded that they have room to dart

* Rowland's great photogiaph of the solar spectrum is on a scale which is about three times larger than that of Angström's map; and, from the exigencies of the case, the lengths of the degrees upon it differ slightly from strip to strip. To adapt a gauge to it, begin by extending both ways the scale of the strip under examination till it.reaches zero in one direction and 10,000 in the other. Orer the 10,000 mark erect a micron, and from the top of it draw the inclined plane to the zero mark. This is the gauge whose ordingtes will be the wave-lengths of the rays represented in that strip of the map. Each strip will require its own gauge; but none of them will be far from 39 metres long, so that they are about three times more acute than the proposed standard gauge. 
to a certain distance along a free path, in the intervals between their encounters with one another; and information as to the average length of these little journeys can be deduced from experiments on the viscosity of gases. If the gas is a tolerably "perfect" one; at the ordinary temperature; and exposed to the pressure of one atmosphere, the average length of the "free path" of the molecules is small. In fact the observed amount of the viscosity assigns to it in air a length equal to the ordinate of our gauge at a distance of something like three quarters of a metre (30 inches) from its apex; and although the mean length of the free path differs from one gas to another, it is in all a magnitude of this order*. Note that this is a good deal smaller than what we have found to be the "minimum visibile."

Witbin the receiver of an air-pump the free path becomes longer, until at the excessive attenuations that $\mathrm{Mr}$. Crookes obtains by working his compound Sprengel-pump for a long time, its average length may even reach to several centimetres, which would be the ordinate of our gauge at a distance from its apex of some hundreds of miles. Ponderable matter is then in what Mr. Crookes calls the radiant state.

2. The average spacing of the molecules in a gas (i.e. their average distance asunder at any one instant of time) may be obtained in various ways †; e.g. it may be deduced from the

* See Philosophiea] Magazine for A ugust 1868, p. 138.

+ Calling the average length of the free path $\lambda$, the average interval between the molecules $\sigma$, and the average "diameter of a molecule" $\delta$; we can obtain $\lambda$ from experiments on viscosity, we get $\delta / \sigma$ from observing the condensation which the gas undergoes when liquetied, and one other equation between $\lambda, \sigma$, and $\delta$ would enable us to obtain all three.

Now it is evident that $\lambda$ (the average length of the journeys of the molecules) will, cateris paribus, increase if $\sigma$ (the space between the molecules) is increased, which may be effected by expanding the gas, and will decrease if $\delta$ (the distance within which the molecules sensibly act on one another) is increased, which may be effected by exchanging one gas for another. It is, in fact, a function of these two quantities and of others, viz. of the velocities of the moleeules (the mean of the squares of which is known from the pressure and density of the gas), of the events that occur in the struggle of two molecules with one another during their brief encounters, and of the time occupied by these struggles.

The events that occur during the encounters and the time they last are not sufficiently known for the actual equation to be set down: but hypotheses can be framed in regard to them-as, for instance, that the molecules when they encounter simply rebound like hard elastic globes - which enable us to ascertain what function $\sigma / \lambda$ would be of $\delta / \sigma$ if the hypothesis were true, and thus enable us to judge what kind of magnitudes $\delta$ and $\sigma$ are.

The quantities $\lambda$ and $\delta$ vary within wide limits from gas to gas; but it is one of the elementary propositions in the kinetic theory of gases that $\sigma$ 
last measure by taking into account the degree in which the gas falls short of being "perfect," $i$. e. of accurately fulfilling the law

$$
\frac{p v}{p^{\prime} v^{\prime}}=\frac{\mathrm{T}}{\mathrm{T}^{\prime}}
$$

where $p$ is pressure, $v$ volume, and $\mathrm{T}$ absolute temperature. Judged in this and other ways, it appears that the average interval between the molecules of any of the more perfect gases, when at atmospheric pressures and temperatures, is something like the ordinate of our gauge at the distance of one centimetre from its apex *. If the vacuum in a Sprengelpump be carried so far as to reduce the pressure to one millionth of an atmosphere (which is not very far from the greatest exhaustion that can be attained), the average spacing

(which may be called the average spacing of the molecules) is nearly the same in all nearly "perfect" gases when compared at the same pressure and temperature. This is, in fact, the truth that underlies and gives its value to Avogadro's erroneous hypothesis that at the same temperature and pressure the size of the gaseous molecriles of all substances is the same. In the present, state of science it is desirable that every practicable effort should be made to determine with more exactness the value of this important physical quantity.

* Phil. Mag. for August 1868, p. 140. If we assume, in conformity with the estimate in the text, that the molecules of a gas at, say, $21^{\circ} \mathrm{C}$. and 760 millim. pressure, are as numerous within a given space as would be a number of points cubically disposed at intervals of a ninethet-metre asunder (this being the ordinate of our gauge at the distance of one centimetre from its apex); then the number of molecules of the gas in every cubic millimetre of its volume is a uno-eighteen-the number represented by 1 with eighteen 0 's after it. Hence, in a litre of the gas there will be a million times more, $i$. e. a uno-twentyfour of molecules. Now at the above-mentioned temperature and pressure a litre of hydrogen weighs just one twelfth of a gramme $\left({ }^{\circ} 083^{\prime}\right)$. Hence the mass of each molecule is the twentyfourthet of this $(i, e$. the fraction represented by 1 in the numerator, and 1 followed by twenty-four 0 's in the denominator), $i$. $e$, it is $=8^{\prime} \cdot 3 \times \times v^{\text {ets }}$ of a gramme; and according to this computation the chemical atom of hydrogen, being the semi-molecule, has as its mass $4^{\prime} \cdot 16 \mathrm{xxvi}^{\text {tet }}$ of a gramme. This is probably somewhere in the neighbourhood of the true value; so that we may regard the mass of a chemical atom of hydrogen as a mass probably not more than a few times more or a few times less than the twenty-fifthet or twenty-sixthet of a gramme. This seems the best approach that can at present be made to estimating the mass of a chemical atom.

The determination depends upon the average spacing of the centres of the molecules of a gas at standard temperature and pressure (see last footnote); and if this very important physical magnitude, which is common to all perfect gases, can be ascertained with more accuracy, we shall get a proportionally better estimate of the mass of a chemical atom. Of course if the mass of the atom of any one element, $e . g$. hydrogen, be determined, the masses of all the others become known by the chemical tables of atomic weights. 
of the molecules will have increased up to being equal to the ordinate of our gauge at a distance of about a metre from the apex. It is instructive to observe that even this enlarged interval is ultra-visible, and that in this so-called extreme vacuum there remain something like a million millions of molecules of the gas in every cubic millimetre of the space within the receiver- $i$. e. about a thousand in every cubic micron.

3 . The magnitude to be next considered is the diameter of a molecule. By this is to be understood the distance within which the centres of two molecules must come, if they are sensibly to deflect each other's path. This size of the gaseous molecule, as it may be called, is intimately related to the ratio of the last two measures to one another, and may be deduced from that ratio. Or it may be obtained by observing the condensation which a gas or vapour undergoes when passing into the state of a liquid or solid. Estimated in either of these ways, it appears to be usually about the 8th, 10th, or 12th of the last measure-that is, it is something like the ordinate of our gauge at a distance of one millimetre from its apex.

\section{Sinaller Magnitudes.}

The diameter of a gaseous molecule, as above defined, is the smallest measurement for which the present gauge is suggested as convenient, as it is also the smallest magnitude of the actual size of which any approximate estimate has been made. But we have, through the spectroscope, indications of important events in nature that are perpetually going on within each gaseous molecule, and probably on a very much smaller scale. For example, an easy calculation will show that the motion within the molecules of sodium to which the principal double line in its spectrum is due $e^{*}$ a motion which is repeated $508,911,000,000,000, i . e$. more than five hundred millions of millions of times, every second within each molecule-would need to have a velocity several times greater than that of the earth in its orbit (which is a velocity of 30 kilometres, 19 miles, per second) if the range of these motions is the whole diameter we have attributed to the molecule. This consideration, though not decisive, is nevertheless quite sufficient foundation on which to base the expectation that, if ever we are able to ascertain the actual range of this motion and others of a like kind, they will turn out to be much smaller than the ordinate of our gauge at a distance of a millimetre from its apex; so that if ever we discover any

* See Stoney on Double Lines, Scientific Transactions of the Royal Dublin Society, vol. iv. p. C03. 
way of quantitatively estimating such events, we shall require another and more acute-angled gange to aid us in appreciating them.

\section{Of the Borderland of the Visible.}

Meanwhile the gauge now proposed will, it is hoped, help the scientific student to obtain a more connected view of nature, by placing before him in somewhat clear evidence the relation in which some of the larger molecular events stand to the dimensions of the smallest objects he can see with his microscope.

He should never forget that even the most minute of these microscopic objects is an immense army of molecules*, or semi-molecules, crowded together, more numerous indeed than all the inhabitants of Europe. The individuals that constitute the battalions are not seen, nor is there the least glimpse of the active motions that are without intermission going on among or within the individuals : nay more, waves of light are too coarse to supply our microscopes with information about the evolutions of the companies, regiments, and brigades of this great army. It is only when the entire army shifts its position that anything can be seen; and my object will be attained if the contrivance I have proposed helps in any degree to bring about a better balance of thought relatively to the cosmos in which we find ourselves : it is so difficult to avoid making the small range of our senses a universal scale with which to measure all nature. Where, for instance, is the justification for our alleging that any visible speck of protoplasm is undifferentiated? And, in fact, are not subsequent events perpetually rebuking this rashness?

A convenient object to help in connecting visible with ultra-visible magnitudes, is the marking on the frustule of the Pleurosigma angulatum (or, Gyrosigma angulatum), one of the commonest of test-objects. The Jittle brown specks are easily seen with the higher powers of a microscope if a good condenser and the proper stop be used, and their distance asunder from centre to centre is somewhere between $\cdot 64$ and . 65 of a micron, according to the best determinations I can make. This is a trifle more than the spacing deduced from Professor Smith's measurement of the interval between the

* That is, of molecules such as are present in the gaseous state of the ultimate chemical constituents of the speck of matter under examination. These in a highly organized substance like protoplasm are assaciated into much larger organic groups, that may be called mega-molecules, and may be likened to the companies or regiments of the brigades and corps that make up the army. 
rows. It is the ordinate of our gauge at about six and a half metres from its end, and is the wave-length of a ray of red light not far from the red hydrogen-line, the line $C$ of the solar spectrum; so that the brown dots succeeding one another in a row mark off in the field of the microscope the successive waves of this particular ray of light.

The dots are arranged in rows parallel to the sides of an equilateral triangle, and with oblique light coming at right angles to any one of these sets of rows, the dots will elongate and almost run into one another in a way that makes the rows look like a ruling of parallel lines. These parallel lines are at shorter distances asunder than the dots in the ratio of $\sqrt{3}$ to 2 , and accordingly present to the eye intervals equal to the wave-length of a green ray less refrangible than the line $\mathrm{E}$ of the solar spectrum. The interval in this case is the ordinate of our gauge at a distance of about five metres and a third from its apex.

Furthermore, what we have found above to be the minimum visibile is a little more than one third of the interval from centre to centre of the dots, or a little less than half the interval of the rows. It is well illustrated by the Pleurosigma markings. In fact, judging from similar markings on other scales, the round dots would be sern as rings were it not for their small size, which prevents the opposite sides of the ring from being seen as two objects. They accordingly look like disks".

\section{Of the Nomenclature of Small Measures.}

It will often be found convenient to connect the proposed standard gauge with another useful way of describing small magnitudes. Let us understand by a sixthet a unit in tie sixth place of decimals, $i$.e. the fraction of $1 / 10^{6}$, and let us use the phrase sixthet-metre, or metre-sixthet, to mean the sixthet of a metre, in the same sense in which we say halfinch or quarter-inch to mean the half or quarter of an inch.

We can then conveniently express the following table of equivalents.

The ordinate of the standard gauge, at a distance :-

Of ten metres from the apex $=$ a sixthet-metre.

Of one metre $", \quad=$ a seventhet-metre.

Of one decimetre " $" \quad$ = an eighthet-metre.

Of one centimetre $\quad,=$ a ninthet-metre.

Of one millinetre $\quad,=$ a tenthet-metre.

* The white pearl-like specks which take the place of the dots when they are a little out of focus, must not be mistaken for their being seen as rings. They are an optical effect, and of larger size

Phil. Mag. S. 5. Vol. 34. No. 210. Nov. 1892. $2 \mathrm{G}$ 
The sixthet-metre is identical with the micron spoken of above. Many writers represent it by the symbol $\mu$.

The ninthet-metre is the thousandth part of a micron. It bas sometimes been called the micro-millimetre, and is by some writers represented by the symbol $\mu \mu$.

The tenthet-metre is the same measure as is usually called the tenth-metre. It has also sometimes been called the tenth-metret.

\section{Of the Smallest Magnitudes that have been Measured.}

The most minute magnitudes that have been actually measured are differences of wave-length. These can be determined with truly astonishing precision by observations with the diffraction-grating spectroscope, so much so that they carry us down to magnitudes that are fractions of the diameter of a gaseous molecule. The observation is most easily mado in the case of close double lines. In these the interval between the two constituents is due to the difference of their wave-lengths, and by measuring the former the latter can be ascertained. Thus, an interval of one degree on Angström's or Rowland's map indicates a difference of wave-length amounting to a tenthet-metre, which, as we know, is the ordinate of our standard gauge at a distance of a millimetre from its end. But lines have been seen to be double with Prof. Rowland's gratings, in which the separation of the two constituents is not more than from $1 / 30$ th to $1 / 100$ th part of a degree. In the latter case the difference of wave-length is only one twelfthet-metre. This is the ordinate of our gauge at a distance from its apex which is little more than the diameter of a single blood-corpuscle, and may be taken to be the smallest measurement that can as yet be directly effected with certainty.

The following is a list of close double lines which $I$ have myself seen in the solar spectrum with a small Rowland's grating * :-

The solar line $b_{3}$, and a multitude of other close doubles, shown as such on Rowland's map (2nd Series, 1888).

The less refrangible of the two $\mathrm{E}$ lines.

The line in the $\mathrm{E}$ group at $\lambda=5264.4$ of Rowland's scale.

* The grating is flat, nearly an inch and three-quarters long, contains about 25,000 lines, and the observations were made in the fifth spectrum. In this spectrum the image is formed by bringing together every fifth wave of light out of a series of 125,000 consecutive waves, and the ruling. nust be sulficieutly accurate te effect this. 
Appreciation of Ultra-visible Quantities.

The nickel line which is nearly midway between the two

$D$ lines; and the line at $\lambda=5892 \cdot 6$. This is the second from the nickel line towards $\mathrm{D}_{2}$, of the elevell lines which are at all times visible between the two $\mathrm{D}$ lines.

The least refrangible constituent of the triple line at $\lambda=5328 \cdot 7$.

This last is about the closest double that my spectroscope will resolve. There is no micrometer on my instrument, so that I cannot give measures, but I estimate the coarsest of these, those first mentioned, to have a difference of wavelength under two eleventhet-metres-and in the closest, that last mentioned, it cannot be more than a very few twelfthetmetres. Most of thein could be measured with a good micrometer.

This can be accomplished with one of the smaller of Professor Rowland's splendid gratings; and he himself and other observers have carried matters farther, by taking photographs with the best of his great six-inch concave gratings. This may give some idea of the marvellous precision of this, the latest and most searching appliance for exploring Nature. By it a brilliant series of discoveries have already been made in stellar astronomy; and we may anticipate still greater achievements from the distance to which it can throw its plumb-line into the obscure depths of molecular events.

\section{Time Relations.}

The fragments of time that can be appreciated with accuracy in this way are even more wonderful in their minuteness than are the differences of length. Time relations, however, lie somewhat outside the scope of the present essay ; but they, too, should be carefully pondered by anyone who wants to know what Nature really is. And after thus taking the best survey that he can, he should bear in mind that all he can do is to gauge the little that man has been fortunate enough to detect; and that far more may lie beyond the ken of any human being than the immense range which now lies within it. He should also reflect that the few molecular events that are already known succeed one another with such astonishing rapidity that the swiftest visible motions are, in relation to them, as sluggish and as gradual in their progress as are the changes in the configurations of the constellations owing to the proper motions of the fixed stars, in their relation to us and to the events we can see occurring about us on the earth. In fact, the thousandth of one second of time is, in relation 
to them, comparable with some such period as twenty or thirty thousand years in its relation to man's slow thoughts, or the driftings about of those accumulations of molecules which are the only kind of objects he can perceive even with the highest powers of his microscope. These visible objects, these arnies of molecules massed together, seem to him sometimes at rest and sometimes in motion; but in either case strenuous activity within and between the molecules themselves never ceases, nor the perpetual response between them and the æether through which they keep up a communication with one another at a distance. The magnitude of the consequences throughout all Nature of this unflagging intercourse between molecules cannot be approached by the utmost thought we can give to it. It is quite impossible for us to appreciate it adequately. The human eye placed anywhere intercepts a small fragment of the messages in their transit, and is thus a detector of their presence. But it does so roughly. It jumbles up the immense detail which even our spectroscopes can show to be included within this fragment. Yet even so, how much our eyes show us wherever we turn them, and with what seems to us such marvellous promptness! The spectroscope in some respects penetrates farther as a detector. Even it, however, fails to reach much detail that we know to be present, e.g. it cannot tell us the innumerable interruptions or the various orientations or the phases of the actual motions. And, at the best, both these detectors together can give us but a very slender notion of the real activity that is going on, and of the precision and fulness with which the molecules everywhere about us are energetically exchanging many millions of different messages with one another every second. Such is Nature as it really is.

\section{On the Bearing of these Determinations on other Branches of Study.}

Determinations such as those dealt with in this paper have a bearing upon almost every study that is occupied either in the interpretation of material Nature, or investigating the relation between the thoughts of animals and the operation 3 that go on in their brains ; inasmuch as the whole of material Nature is found, on careful analysis, to rest on moleculeson their mutual relations and motions, on the events going on within the molecules, and on those which they excite in the medium in which they move.

1. One example of this influence upon other studies is the general limitation which molecular determinations impose 
upon the methods employed in dynamical inquiries, as pointed out in a Paper by the present author on "Texture in Media." [Scientific Proceedings of the Royal Dublin Society, vol. vi. p. 392 ; Phil. Mag. for June 1890, p. 467.]

2. The direct bearing of the inquiry upon chemistry is obvious. It is briefly referred to in the Scientific Transactions of the R. D. Society, vol. iv. p. 608 . In fact the record of the chemist is not unlike what one often sees upon a tombstone-"Born in such a year; Died in such another ;"while the intervening life is passed over in silence. So the chemist submits two or more substances to their mutual influence, and finds that such and such substances emerge; but he takes little note of the eventful time during which all the protracted contests of the reaction have taken place, which, if it has lasted for only the five-hundred-thousandth of one second, has been as long in reference to the activities of the molecules as a long life of 60 years would be in reference to all the thoughts and actions of a man.

3. The minimum visibile, as defined above, is between the fourth and fifth of a micron, and a speck whose volume is the cube of this may be regarded as the smallest organic speck that the biologist can distinguish from other specks by the highest powers of his microscope. Its volume is accordingly about one-hundredth of a cubic micron-about the 1/7000th part of the volume of one blood-corpuscle. Now, liquid or solid material, if resolved into its chemical elements, and if these be brought into the gaseous state, will, at the temperature and pressure of the atmosphere, expand about 1000 times. Hence the foregoing speck, if thus resolved into gas, would occupy about ten cubic microns. But this volume of gas at that temperature and pressure contains about a uno-ten $(10,000,000,000)$ of molecules, which for the most part will consist each of two chemical atoms. Hence the number of chemical atoms in our speck may be taken to be about two uno-tens. Our speck, perbaps, consists of very complex organic molecules; but however complex each of these may be their number must nevertheless be very great. For, let us make the liberal allowance of 2000 chemical atoms for each organic molecule, and the number of these very complex molecules will be about ten millions. This is an army quite large enough to admit of an immense amount of differentiation within its ranks - of very active operations within and among the complex molecules or between brigades of them-all of which are ultra-visible events. These are facts which overy biologist should keep constantly before his mind when carrying out his investigations and interpreting them, and especially 
when he is tempted either to speak or think of " undifferentiated protoplasm."

4. A still more striking instance is presented when we consider the operations of the human mind. Here I will make the usual assumption, that every perception or other thought in the mind is accompanied by a physical event occurring in the brain, which is connected with it in such a way that neither presents itself without the other. Of this event we know that it is of a kind that arises only in living brains and in them only while the man is either awake or dreaming. We also know that it is of a kind that lasts for a considerable time when it does occur, viz. throughout the duration of the perception or other thought in the mind.

This last consideration is very significant. The event in the brain with which human perception or any other human thought is associated must be one which can last while the thought lasts, i.e. for a time immensely long when compared with the original molecular events that are going on. The event may, for example, be such an event as a strain consequent on a stress, whether dynamical or electromagnetic, acting on some part of the brain; or it may be of the nature of a forced vibration or current. These are events which would continue in existence so long as the stress is applied, and will cease when the stress is removed: they fulfil the requisite time conditions. Another event which would fulfil the time conditions is an undulation-dynamical, electromagnetic, or of any other kind. The waves that make up an undulation may continue in it but a short time, some passing off while others come on, and the motions or stresses of which each wave consists may be such as succeed each other with extreme rapidity, while all the time the undulation viewed as a whole continues as much unchanged as a human thought does while it lasts. Hence an event of this kind may, so far as its relation to time is concerned, be that event in the brain which is intimately associated with human thought. Possibly the event we are in search of may be found among the processes of metabolism whereby nutrient matter brought by the blood becomes part of the brain; or more probably among those processes in which matter that had formed part of the brain separates and is swept away either by the blood or lymphatic vessels. Events of this kind, including every interference with or modification of those here specified, and the many other events which like them may be described as stream effects, are marked by the peculiarity that a vast number of molecules are concerned in them in such a way that different molecules successively take up the running. 
All such events fulfil the necessary condition of continuing temporarily in existence, as each of our thoughts does, for a time which may be immensely long compared with the fundamental events within or between the molecules, or in the interfused æther. It must, however, be borne in mind that it is upon these fundamental events that the whole superstructure rests; and that stream effects are in relation to them of the nature of very small outstanding residual events which remain over, when the rest-the great bulk of the events that are actually happening-are such as balance one another* .

It is evident that it is among protracted events such as those spoken of in the last paragraph that we must search for the physical event in the brain with which human thought is associated. It is also evident that it is with but a very small selection out of the vast number of such events occurring in the brain during life, that the thoughts of which man is conscions can be directly associated. All the rest of the innumerable stream events, and all the underlying fundamental ovents within and between the individual molecules, go on besides.

Now, what happens in the brain is an index to us of what is going on in that portion of the Autic Universe $f$ which is most closely connected with human thought. For what goes on in the phenomenal world (to which the brain belongs) is an index to us of what is going on in the Autic Universe (to which our thoughts belong), in the same sense in which a weather-cock is an index to us of the direction of the wind; since what occurs in the phenomenal world is dependent upon and determined by what occurs in the Autic Universe (see passim a paper by the Anthor, "On the Relation between Natural Science and Ontology," Scientific Proceedings of the Royal Dablin Society, vol. vi. p. 475). Hence in the Autic Universe there are events as closely related to the thoughts which exist in my mind at any time, as are the other physical events going on in my brain at that time to those few which are directly associated with my thoughts. The autic events here spoken of probably more or less distantly resemble the events within my own mind of which I am conscious, inasmuch as all the physical events in the brain in a certain

* Wind is such a residual event. It arises whenever there is a small preponderance in one direction of the very much swifter velocities with which the individual molecules of air are at all times darting about in all directions. So also the current in a river is a similar residual event.

$\dagger$ Auta, actual existences (of which the thoughts that are in my mind are a sumple directly known to $\mathrm{me}$ ). The Autic Uuiverse, the totality of all really existing things, of all auta. 
degree resemble one another; and, in particular, the time relations between them and between them and my thoughts are the same as the time relations of the physical events that, as a consequence, go on in my brain. This body of events in the Autic Universe and the thoughts or other auta between which they occur, may suitably be spoken of as a synergos (бvveprós, a fellow-worker) which is ever on the alert to work along with my mind, and on which the thoughts that are my mind as much depend (in the autic sense of that word), as do those few physical events in my brain which are associated with my thoughts depend (in the physical sense of the word) upon the vast multitude of other physical events also going on in my brain. The one cannot even exist without the other.

The lesson to be learned from all this is that psychology and the other branches of metaphysics, as presented by the ablest men who were unaware of the existence of this synergos and of the large degree in which it intervenes in all that happens in the mind, will now have to be rewritten. In memory ; in the association of ideas ; and in the other miscalled "faculties and operations of the human mind," it is little we do: it is much that is done for us*. Man's mind-

* Take, for example, some particular instance of menory. I remember where $I$ sat at breakfast this morning, where my companions at, also several particulars of what was said during breaffasi, of the gestures of my companions, of my own motions, of what I ate, of the equipage on the table, and so on. None of these things have occupied my thoughts since breakfast, till I sat down to write these lines; since when they have all come into my mind.

Now what does all this mean? It means that the group of thoughts which I call myself-my mind-and which at each instant is a group the several parts of which are connected and interacting in that way that we call being within one consciousness, is a group of thoughts that has undergone change; that one instance of this change has been the discontinuance of the above-recited perceptions that formed part of the group at breakfast-time, and that another instance has been the occurrence now within this group of what is more or less an imperfect and much modified repetition of some of those perceptions, accompanied by the add'tional thought which we call being aware that they had at breakfasttime occupied a place within the group in the fuller form of complete perceptions. During the intervening hours none of these occupied any place in the group either in their fuller or in their modified form: neverthe? ess, there nust have been, somewhere in the autic universe, a chain of causation connecting the original perceptions and the memory of them. Of this chain the first link was a part of my mind, the last link is a part of my mind, but the connecting links have been in the syrergos.

This becomes clearer when we turn to the objective or phenomenal world, which is a kind of shadow thrown in a special way by the succession of events that occur in the autic universe (see Stoney "On the Relation between Science and Ontology, Scientific Proceedings of the Royal Dublin Society, vol. vi. pp. 502,503, 504). My thoughts, which 
the little changeful group of interwoven thoughts that is himself-is a very small part of the great Autic Universe. We must shift our centre, and exchange the metaphysician's narrow Ptolemaic for a broad Copernican view of existence.

\section{The Law of Error and Correlated Averages. By Professor F. Y. EDGEWORTH, M.A., D.C.L.*}

THIS is a contribution to the investigation of the most 1 general conditions under which the exponential law of error is fulfilled; together with some applications to the theory of "correlated averages" $\dagger$.

I. The simplest case in which a group of measurable objects range in conformity with the law of error is where every member of the group is a sum of a certain number of items, or elements, each of which has or has not a certain quality, e.g. the colour white, or, as it may be expressed, assumes the value 1 or 0 , with a certain average frequency (e.g. 1 just as oftenor half as often-as 0 , in the long run) and at random, or in

are a part of the autic universe, are shadowed by certain objective changes in my brain; and the term synergos means that other portion of the autic universe which is shadowed by all the other events that happen objectively in my brain. It appears from physical considerations that the particular stream effects or other changes in the brain that were the shadows of the perceptions I had at breakfast-time, cannot have occurred alone, but were accompanied by more subtile motious or changes in the brain, which were the shadow of, and thus betokened, certain closely associated events then going on in my synergos. These again were succeeded by motions, changes, or states of strain in the brain during the intervening hours, all of which were a part of the varying shadow of the synergos as it underwent whatever changes took place in it during that interval. Moreover, these intervening events in the brain were of such a kind, as the result has proved, that they have been now followed up by motions or changes in the brain which resemble those that were the shadow of my thoughts at breakfast-time, and which are a part of the group of events now going on in wy brain that are the shadow of those thoughts that constitute my mind as it exists at present.

Softening of the brain is the shadow cast within the objective world when very unfortunate events have happened in the autic universeevents which have included a weakening of the power which the synergos and the mind previously had of mutually acting on one another, or else which have prevented the full formation within the synergos of some of the intermediate links of causation spoken of above. Either of these would involve a partial loss of memory.

* Communicated by the Author.

+ See Galton (Proc. Roy. Soc. 1888), "Co-relations and their Measurements;" and Weldon (Proc. Roy. Soc. 1802), "Certain Correlated Variations in Crangon vulgaris;" also "Correlated Aveiages," by the present writer in the Philosophical Magazine for A ugust 1892. 